Interview

\title{
Der Radiologie ein neues Gesicht geben
}

\author{
Im Rahmen des RadiologieKongressRuhr 2013 fand die Mitglie- \\ derversammlung der Rheinisch-Westfälischen Röntgengesell- \\ schaft (RWRG) statt, bei der turnusmäßig ein neuer Vorstand \\ gewählt wurde. In den vergangenen zwei Jahren stand die Ge- \\ sellschaft unter der Leitung des Westfalen Prof. Dr. Wolf-Dieter \\ Reinbold aus Minden. Neue Präsidentin ist Univ.-Prof. Dr. Chris- \\ tiane Kuhl, Direktorin der Klinik für Diagnostische und Inter- \\ ventionelle Radiologie am Universitätsklinikum Aachen (UKA). \\ RadiologieReportRuhr befragte Prof. Kuhl über ihre Erwartun- \\ gen und Intentionen mit Blick auf die neuen Aufgaben.
}

Prof. Kuhl, Sie sind zur 1. Präsidentin der RWRG gewählt worden. Was hat Sie dazu bewogen, für dieses Amt zu kandidieren?

Kuhl: Da die Rheinisch-Westfälische Röntgengesellschaft das Amt paritätisch besetzt, der Präsident also im Wechsel mal aus dem Rheinland, mal aus Westfalen stammen sollte, und abwechselnd aus einer akademischen und nicht universitären Einrichtung, wurde ich um die Bewerbung gebeten. Denn für die kommende Periode ist wieder ein Rheinländer mit universitärem Hintergrund an der Reihe. An dieser Stelle möchte ich daher Prof. Reinbold für das Vertrauen danken, das mit dieser Bitte verbunden ist. Ich freue mich darauf, in den nächsten 2 Jahren im gemeinsamen Dialog mit den Kollegen unser Fach weiter voranzubringen.

Welche Ziele möchten Sie als Präsidentin der RWRG verfolgen? Wie könnte das Motto Ihrer Präsidentschaft lauten?

Kuhl: Meine Vorstellung von einer klinischen Radiologie, wie wir sie am Universitätsklinikum Aachen auch leben und verfolgen, ist eine - wie ich finde - durchaus zukunftsweisende. Jeder weiß, dass der Anteil der Radiologie an der Behandlung eines Patienten sehr groß ist und tatsächlich immer weiter wächst. Dennoch ist es vielerorts so, dass die Radiologie eher an Boden verliert und aus dem Bewusstsein der Patienten - und manchmal auch unserer Zuweiser - eher schwindet. Vor ein paar Jahren lautete eine Kampagne des ACR und der RSNA daher „Give radiology a face!“. Damit sollte diesem Trend entgegengewirkt werden. Aber die Errungenschaften der Teleradiologie, die Hochdurchsatzverfahren der modernen Schnittbilddiagnostik wie auch die besondere Abrechnungsweise des DRG-Systems bedingen geradezu, dass der Radiologe aus der Wahrnehmung der Patienten zusehends verschwindet und im Wesentlichen als interner Dienstleister fungiert, der genau das abarbeitet, was auf Anforderungsscheinen steht. Das kann dann in den outgesourcten Radiologen münden, der am anderen Ende der Welt sitzt und Bilder befundet. Sicher nicht das, was wir wollen. In Aachen versuchen wir, diesem Trend etwas entgegenzusetzen. Wir sind für die Patienten sichtbar. Zum Beispiel, weil wir mit ihnen sprechen und uns persönlich kümmern. Das ist nicht immer bequem für uns, wird aber sowohl von den Patienten als auch von denen, die uns Patienten zur Untersuchung oder Behand-

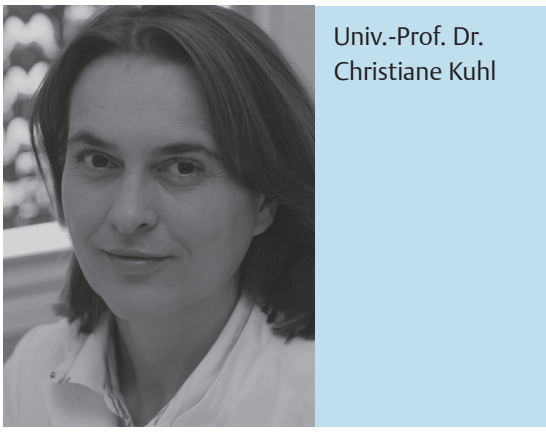

Im Profil

Seit 2010 leitet Prof. Dr. Christiane Kuhl die Klinik für Diagnostische und Interventionelle Radiologie im Universitätsklinikum Aachen. Die 1966 in Bonn geborene Radiologin ist vor allem für ihre Forschung auf dem Gebiet der MRT-Diagnostik von Krebserkrankungen mit Schwerpunkt Brustkrebsdiagnostik bekannt. Für ihre Arbeiten erhielt sie zahlreiche nationale und internationale Auszeichnungen. Weniger bekannt ist, dass sie zwischen 2004 und 2010, also bis zu ihrer Berufung nach Aachen, Deutschlands erste und einzige Schwerpunktprofessur für Onkologische Diagnostik und Interventionelle Tumortherapie innehatte. Einen Ruf auf eine ordentliche Professur für Brustdiagnostik an die Harvard-Universität in Boston, USA, lehnte sie 2007 ab, um sich auch künftig mit der Radiologie in ihrer gesamten Breite beschäftigen zu können.

lung schicken, sehr geschätzt. So sind Auseinandersetzungen darüber, wann ein $\mathrm{Pa}$ tient welche Untersuchung benötigt, wesentlich weniger virulent und drängelnde Zuweiser eher die Ausnahme und nicht die Regel. Mein Appell lautet deshalb: Liebe Kollegen, Ihr arbeitet nicht für Eure Zuweiser, sondern für gemeinsame $\mathrm{Pa}$ tienten. Und Ihr habt sehr viel beizutragen - nicht nur, indem Ihr Diagnosen stellt. Sondern auch, weil Ihr aufgrund Eurer breiten Ausbildung wirklich gute Kliniker sein könnt. Und so stehe ich für eine selbstbewusste Radiologie, die sich einmischt. Natürlich gibt es in fast allen ra- 
diologischen Fachabteilungen Arbeitsbereiche, in denen es einfach nur darum geht, eine große Zahl von Aufnahmen oder Röntgenbildern wegzubefunden. Aber darin darf sich unser Fach nicht erschöpfen. Entscheidend ist, dass wir Radiologen Patientenverantwortung übernehmen oder zumindest mittragen. Dann wird man von Patienten als Arzt und von zuweisenden Fächern als gleichberechtigter Kliniker wahrgenommen.

Gibt es Bereiche in der Radiologie, in denen dieses Verhalten bereits realisiert wird?

Kuhl: Ja, schon. So zum Beispiel in der Mammadiagnostik - eine Spezialität, die ja gern mal eher belächelt wird. De facto läuft in der Mammadiagnostik aber vieles, was auch für den Rest der Radiologie paradigmatisch sein könnte. Dazu gehören die standardisierte Beschreibung des Befunds nach BI-RADS, die Codierung der Sicherheit der Untersuchungsauswertung nach ACR - aber insbesondere die Übernahme von direkter Verantwortung für den Patienten. In der Mammadiagnostik ist es in aller Regel der Radiologe, der die Ergebnisse der Untersuchungen mit der Frau direkt bespricht. Der Radiologe allein entscheidet über die Notwendigkeit einer Biopsie, führt sie selbst durch und bespricht das Ergebnis mit der Patientin und ihren Angehörigen. Oft genug sucht er sogar den passenden Gynäkologen für die Patientin, womit er seinerseits zum Zuweiser wird. Dieser enge Kontakt zum Patienten und zu den Kollegen ist sicher auch in anderen Bereichen der Radiologie möglich - das ist zumindest die Erfahrung, die wir in Aachen machen.

Wie sehen Sie die Rolle der Radiologie und die interdisziplinäre Zusammenarbeit am UKA?

Kuhl: Eine disziplinübergreifende Zusammenarbeit kann nicht durch Vorschriften verordnet werden - ihr Gelingen hängt immer an den beteiligten Menschen. Zudem setzt Interdisziplinarität grundsätzlich voraus, dass es überhaupt Disziplinen gibt - also exzellente fachliche Expertise auf bestimmten Sektoren. Für wirkliche interdisziplinäre Zusammenarbeit müssen sich die verschiedenen Disziplinen jeweils gegenseitig vorbehaltlos anerkennen. Heißt für uns: Die anderen Fächer müssen anerkennen, dass der Radiologe als Experte in Sachen bildgebender Diagnostik und bildgesteuerter Therapie die damit verbundenen Entscheidungen treffen muss - und nicht etwa nur als ausführendes Organ die Entscheidungen Dritter abarbeitet. Erfreulicherweise sind die Direktoren der anderen Abteilungen des UKA einer klinisch aktiv agierenden Radiologie gegenüber sehr aufgeschlossen. So nehmen bei uns nach Möglichkeit an den Haupt-Tumorkonferenzen gleich2 Radiologen teil: einer, der die Bilddemonstration vorbereitet, und ein 2 ., der auf der Seite der Therapeuten sitzt. Letzterer diskutiert und entscheidet gleichberechtigt mit über die Therapie des Patienten. Am UKA hält die Radiologie ein eigenes Bettenbelegungsrecht und führt Patienten eigenverantwortlich. Außerdem haben wir eine eigene Ambulanz als Anlaufstelle für Patienten geschaffen, die uns zur Behandlung vorgestellt werden. Wie alle anderen Fachkliniken auch möchten wir die Patienten selbst klinisch ansehen und alle für die Konzipierung eines radiologisch-interventionellen Behandlungsplans notwendigen Informationen zusammenführen, bevor wir die finale Therapie-Entscheidung fällen und die Patienten einbestellen. Erstaunlicherweise ist das aber ausgerechnet in der Radiologie eine offenbar sehr seltene Praxis - und das, obwohl wir doch mit teils sehr komplexen und sehr differenzierten, individuellen therapeutischen Vorgehensweisen arbeiten. In dieser Ambulanz finden auch die Gespräche mit den Patienten und ihren Angehörigen statt - vor den Behandlungen, wenn es darum geht zu erklären, was das Ziel und die Erfolgsaussichten der Therapie sind, aber auch nach der Behandlung, wenn wir die Patienten zur Verlaufskontrolle einbestellen. Letzteres geschieht regelmäßig nach festgelegtem Protokoll. Denn auch hier sind wir selbst doch diejenigen, die am besten entscheiden können, ob oder ob nicht zum Beispiel nach einer TACE noch einmal nachgelegt werden muss. Vorab werden die Patienten dann von uns in der Tumorkonferenz vorgestellt. Denn wenn wir Interdisziplinarität einfordern, müssen wir uns auch selbst daran halten und nicht einfach allein drauflosbehandeln. All das ist anstrengend und gelingt nicht immer und nicht immer reibungslos - aber insgesamt glaube ich, dass wir in Aachen auf einem guten Weg sind. Der enorme Zulauf, den die Radiologie am UKA hat, ist für uns jedenfalls eine gute Bestätigung.
Hat es für Sie eine Bedeutung, die 1. Frau an der Spitze der RWRG zu sein, und würden Sie sich selbst als Quotenfrau bezeichnen?

Kuhl: Na ja. Die Frage hatte ich früher erwartet (lacht). Niemand ist gern Quotenfrau, weil es die eigene Qualifikation implizit infrage stellt. Es ist aber ja offenkundig, dass Frauen in den verschiedenen Gremien unserer radiologischen Fachgesellschaft - von der Arbeitsebene der AGVorstände bis ins Präsidium - deutlich unterrepräsentiert sind. Das ist nicht nur aus den hinlänglich bekannten sogenannten Gender-Aspekten problematisch. Sondern es sind schließlich diese Gremien, in denen die klinische und akademische Praxis unseres Fachgebiets geprägt und vorangebracht wird. Und hieran sind Frauen - fast wie zu Conrad Röntgens Zeiten - unverändert kaum beteiligt. Ich erlebe es täglich, dass extrem gute Kolleginnen in Oberarztpositionen verunsichert sind, weil sie nicht wissen, ob sie wirklich eine Führungsposition anstreben sollen. Weil es keine Vorbilder gibt. Weil die jungen Frauen kaum Klinikdirektorinnen oder Chefärztinnen sehen, an deren Lebensweg oder Auftreten sie erkennen können, ob eine solche Position das Richtige für sie persönlich sein könnte. Junge Frauen oder Frauen allgemein - haben andere Vorstellungen von dem, was ihnen im Leben wichtig ist. Es ist deshalb wesentlich, dass es mehr Frauen gibt, die ihnen zeigen, dass die Leitung einer Klinik oder einer Fachgesellschaft persönlich erfüllend sein kann und sie keineswegs ein patientenfernes oder gar einsames Dasein fristen müssen. Ganz im Gegenteil, möchte ich sagen. Als Klinikdirektorin habe ich die Möglichkeit, nicht nur Patienten zu betreuen, sondern junge Menschen für das zu begeistern, was mir selbst am Herzen liegt. Sie ein Stück ihres Lebenswegs zu begleiten. Zu sehen, wie sie sich entwickeln, wie sie mit ihren Aufgaben wachsen. Für die man sich freut, wenn ihnen etwas gelingt nicht nur beruflich, sondern auch im privaten Leben. Das erfüllt mich mit großer Zufriedenheit.

Und ob das nun populär ist oder nicht: Ich bin aus diesen Gründen davon überzeugt, dass sich hierzulande nichts ändern wird, wenn wir nicht vorübergehend (!) mit einer Quote arbeiten, um den Anteil von Frauen in Führungspositionen zu erhöhen. Auch und zuvorderst in unserer eigenen Fachgesellschaft. 